\title{
Fire-mediated disruptive selection can explain the reseeder- resprouter dichotomy in Mediterranean-type vegetation
}

\author{
Res Altwegg • Helen M. De Klerk • Guy F. Midgley
}

Received: 12 December 2013 / Accepted: 7 October 2014

(C) Springer-Verlag Berlin Heidelberg 2014

\begin{abstract}
Crown fire is a key selective pressure in Mediterranean-type plant communities. Adaptive responses to fire regimes involve trade-offs between investment for persistence (fire survival and resprouting) and reproduction (fire mortality, fast growth to reproductive maturity, and reseeding) as investments that enhance adult survival lower growth and reproductive rates. Southern hemisphere Mediterranean-type ecosystems are dominated by species with either endogenous regeneration from adult resprouting or fire-triggered seedling recruitment. Specifically, on nutrient-poor soils, these are either resprouting or reseeding
\end{abstract}

Communicated by Jennifer A. Lau.

Electronic supplementary material The online version of this article (doi:10.1007/s00442-014-3112-6) contains supplementary material, which is available to authorized users.

R. Altwegg

South African National Biodiversity Institute, P/Bag X7,

Claremont 7735, South Africa

R. Altwegg $(\square)$

Statistics in Ecology, Environment and Conservation,

Department of Statistical Sciences, University of Cape Town,

Rondebosch 7701, South Africa

e-mail: res.altwegg@gmail.com

R. Altwegg

African Climate and Development Initiative, University of Cape

Town, Rondebosch 7701, South Africa

H. M. De Klerk

Department Geography and Environmental Studies, Stellenbosch

University, Private Bag X1, Matieland 7602, South Africa

G. F. Midgley

Department of Botany and Zoology, Stellenbosch University,

Private Bag X1, Matieland 7602, South Africa life histories, with few intermediate forms, despite the fact that the transition between strategies is evolutionarily labile. How did this strong dichotomy evolve? We address this question by developing a stochastic demographic model to assess determinants of relative fitness of reseeders, resprouters and hypothetical intermediate forms. The model was parameterised using published demographic data from South African protea species and run over various relevant fire regime parameters facets. At intermediate fire return intervals, trade-offs between investment in growth versus fire resilience can cause fitness to peak at either of the extremes of the reseeder-resprouter continuum, especially when assuming realistic non-linear shapes for these trade-offs. Under these circumstances, the fitness landscape exhibits a saddle which could lead to disruptive selection. The fitness gradient between the peaks was shallow, which may explain why this life-history trait is phylogenetically labile. Resprouters had maximum fitness at shorter fire-return intervals than reseeders. The model suggests that a strong dichotomy in fire survival strategy depends on a non-linear trade-off between growth and fire persistence traits.

Keywords Demography $\cdot$ Fire-return interval $\cdot$ Protea . Stochastic matrix population model $\cdot$ Fynbos

\section{Introduction}

Fitness trade-offs, where a fitness gain due to a change in one trait is opposed by a fitness loss due to a concomitant change in another trait, are a key concept in life-history theory (Stearns 1992). The evolution of fitness trade-offs is still poorly understood (Roff and Fairbairn 2007), but cases where trade-offs lead to the coexistence of distinct 
life-history strategies offer particularly promising opportunities for study. One such case is found in fire-prone plant communities (Bond and Keeley 2005; Keeley et al. 2012), where woody plants have evolved two distinct strategies to cope with fire (Le Maitre 1992; Bond and Midgley 2001; Pausas et al. 2004; Higgins et al. 2008; Pratt et al. 2012). They either have well-protected buds that allow adults to resprout after a fire, or adults are killed by fire and rely solely on their seeds to re-establish in the burnt area (Bond and Midgley 2001; Keeley et al. 2012). This life-history dichotomy is found in most major families of woody plants that dominate Mediterranean-type regions with regular stand-replacing crown fires (Vesk and Westoby 2004), and the two strategies are particularly dominant on the nutrient-poor soils of the South African fynbos and Australian kongwan (Wells 1969; Le Maitre and Midgley 1992; Ojeda 1998; Keeley et al. 2012). Intermediate forms, i.e. species in which some individuals exhibit one strategy and some the other, are rare (Schutte et al. 1995). The clear life-history dichotomy and its appearance in many families raise the question of the evolutionary origin of this dichotomy. Apparently, the trait has been evolutionary labile and repeatedly subject to disruptive selection (Lamont et al. 2011). For example, in the protea genus Leucadendron, the two fire-response strategies are not phylogenetically clustered, suggesting multiple switches between the two strategies within this single genus alone (Barker et al. 2004).

We examined the role of various aspects of fire regime, principally fire frequency or fire-return interval, but also fire season and fire intensity, or extreme events, in shaping the fitness landscape of reseeders and resprouters to see under what conditions disruptive selection may be expected. We refer to reseeder as the strategy that involves all adults dying in a fire and reproduction taking place exclusively through seeds that germinate after the fire. This strategy is also known as 'non-sprouter' (Bond and Midgley 1995; Schutte et al. 1995; Pratt et al. 2012), 'non-persistent semelparous' (Higgins et al. 2008) and 'obligate seeder' (Pausas et al. 2004; Keeley et al. 2012). We refer to resprouter as the strategy where most adults survive a fire and resprout afterwards, but where fire also triggers reproduction from seeds. This strategy has also been called 'persistent iteroparous' (Higgins et al. 2008), 'facultative sprouter' (Pratt et al. 2012) or 'facultative seeder' (Keeley et al. 2012).

Both resprouting and reseeding have advantages (Le Maitre and Midgley 1992; Schutte et al. 1995; Vlok and Yeaton 1999; Bond and Midgley 2003). Initially after a fire, resprouters have safe underground resources from which to draw and can rapidly take advantage of the recently opened spaces, while reseeders must first germinate and establish. At this stage, reseeders require environmental conditions favourable for germination and need predictable rainfall after the fire season to facilitate establishment (Keeley et al.
2012). Later in post-fire succession, reseeders likely incur lower costs of investing in and maintaining carbon-rich defensive structures such as bark, buds (particularly basal buds that are well insulated) and storage tissues (particularly lignotubers) required to support post-fire shoot growth (Pausas et al. 2004; Pratt et al. 2012). Even though the generality of this trade-off is not well established (Bond and Midgley 2003), there is evidence that seedlings of reseeders tend to grow faster than seedlings of resprouters (Pausas et al. 2004; Lamont et al. 2011), and the latter also tend to show slower shoot development (associated with greater root development tied to drought avoidance; Pausas et al. 2004). In the fynbos and kongwan, which experience more pronounced summer drought than other Mediterraneantype regions, reseeders appear to have higher seedling survival than resprouters during the summer drought (Ojeda et al. 2005; Keeley et al. 2012). Reseeders may produce more seeds than resprouters of similar age (Pausas et al. 2004; Ojeda et al. 2005), although this difference mostly disappears when correcting for canopy volume (Bond and Midgley 2003).

With these varied advantages for each life-history strategy, it is not obvious why intermediate forms combining beneficial traits from both strategies hardly ever reach dominance. Indeed, the co-existence of the two strategies is much better investigated (Keeley and Zedler 1978; Enright et al. 1998a, b; Vesk and Westoby 2004; Ojeda et al. 2005; Higgins et al. 2008) than the poorly understood issue of how selective pressures have apparently routinely led to disruptive selection in fire survival strategies. Here, we use demographic estimates obtained from the literature on two fynbos Protea species and a demographic model that uses a set of conceptual trade-offs to examine how fire frequency interacts with basic life-history trade-offs to favour either the reseeder or the resprouter life history, as opposed to hypothetical intermediate strategies. Effects of fire season and fire intensity are also modelled. We examine the tradeoff between fire mortality and growth mediated by different levels of investment in either protective tissue or structural growth. We envision intermediate strategies that invest into some protection at the cost of reduced growth but still suffer substantial fire mortality.

The three main questions that we address are:

1. Does the fire-return interval affect which strategy has higher fitness?

2. Do reseeders and resprouters have higher fitness than intermediate strategies?

3. Does the shape of the underlying life-history trade-offs affect the fitness landscape?

The fynbos vegetation of the Cape Floristic Region (CFR) is one of the most diverse amongst temperate floras 
Table 1 Parameter values selected for modeling demography of reseeding and resprouting proteas, and literature values for selected species on which these were based

\begin{tabular}{|c|c|c|c|c|c|c|c|c|}
\hline & \multicolumn{8}{|c|}{ Model parameter value Literature values } \\
\hline & Reseeder & Resprouter & Protea neriifolia & $n$ & Protea repens & $n$ & Protea nitida & $n$ \\
\hline Seed survival in seed bank $\left(s_{1}\right)$ & 0.4 & 0.4 & & & $0.28-0.4$ & 40,350 & & \\
\hline Seedling survival $\left(s_{\mathrm{s}}\right)$ & 0.05 & 0.05 & & & 0.054 & 40 & & \\
\hline Survival vegetatives $\left(s_{2}\right)$ & 0.95 & 0.95 & $0.6-0.98$ & na & & & $0.919-0.989$ & 54,160 \\
\hline Survival small adults $\left(s_{3}\right)$ & 0.98 & 0.98 & $0.6-0.98$ & na & & & 0.995 & 18,54 \\
\hline Survival medium adults $\left(s_{4}\right)$ & 0.98 & 0.98 & $0.6-0.98$ & na & & & $0.97-0.98$ & 32,123 \\
\hline Survival large adults $\left(s_{5}\right)$ & 0.98 & 0.98 & $0.6-0.98$ & na & & & 1 & 15,22 \\
\hline $\begin{array}{l}\text { Growth from vegetative to small } \\
\text { adult }\left(g_{23}\right)\end{array}$ & 0.2 & 0.06 & $0.15-0.25$ & na & & & $0.04-0.08$ & na \\
\hline $\begin{array}{l}\text { Growth from small to medium adult } \\
\left(g_{34}\right)\end{array}$ & 0.5 & 0.25 & & & & & $0.06-0.25$ & 18,22 \\
\hline $\begin{array}{l}\text { Growth from medium to large adult } \\
\left(g_{45}\right)\end{array}$ & 0.4 & 0.1 & & & & & $0.03-0.06$ & 123,32 \\
\hline Fecundity small adults $\left(f_{3}\right)$ & 15 & 15 & $9-53.4$ & na, 74 & & & $2.6-15.2$ & na, 119 \\
\hline Fecundity medium adults $\left(f_{4}\right)$ & 40 & 40 & $9-34.3$ & na, 30 & & & & \\
\hline Fecundity large adults $\left(f_{5}\right)$ & 80 & 80 & 9-99.4 & na, 160 & $54-1,553$ & na, 40 & & \\
\hline Germination rate after fire $(h)$ & 0.98 & 0.98 & & & & & & \\
\hline Germination rate no fire $(h)$ & 0 & 0 & & & & & & \\
\hline Fire mortality vegetatives $\left(m_{2}\right)$ & 1 & 0.9 & & & & & $0.13-1$ & $116,103,41,38$ \\
\hline Fire mortality small adults $\left(m_{3}\right)$ & 1 & 0.3 & & & & & $0-0.37$ & 29,19 \\
\hline Fire mortality medium adults $\left(m_{4}\right)$ & 1 & 0.2 & & & & & $0-0.29$ & 68,4929 \\
\hline Fire mortality large adults $\left(m_{5}\right)$ & 1 & 0.1 & & & & & $0-0.14$ & $48,43,40$ \\
\hline $\begin{array}{l}\text { Probability being reset during fire: } \\
\text { medium adults }\left(r_{4}\right)\end{array}$ & & 0.5 & & & & & $0.34-0.93$ & $68,46,70$ \\
\hline $\begin{array}{l}\text { Probability being reset during fire: } \\
\text { large adults }\left(r_{5}\right)\end{array}$ & & 0.5 & & & & & $0.17-1$ & $48,37,40$ \\
\hline
\end{tabular}

Abbreviations used in Eq. 2 are given in parentheses. $n$ is sample size on which the estimates were based in the original study, if available ( $n a$ not available). Estimates for P. neriifolia (a reseeder) are from Le Maitre (1987, 1992), and Le Maitre and Midgley (1992), and estimates for P. nitida (a resprouter) are from Le Maitre (1992); and estimates for P. repens (a reseeder) are from Coetzee and Giliomee (1987), Le Maitre (1987), and Musil (1991). Survival rates used in the model were at the higher end of reported values because sample sizes were larger for these studies. Size classes were defined to reflect difference in fecundity. Because of uncertainty in parameter estimates, we examine three alternative parameterisations of the model (Online Resource 1)

(Takhtajan 1986; Cowling et al. 1992; Goldblatt and Manning 2002). One of the determinants of this diversity is thought to be the occurrence of fire (Cowling 1987; Linder 2003; Barraclough 2006; Lamont et al. 2011), which has an intermediate frequency in relation to global ecosystems (return interval of roughly 7-25 years, compared to century time scale in forests and annual to biennial time scale in grasslands and savannas). The dry summer season when fires naturally happen are followed by predictably wet winters, particularly in the west where diversity is greatest (Cowling 1992). Human modification (mainly acceleration) of the fire cycle is thought to have occurred in the past few hundred years, and this may accelerate further under climate change scenarios (Wilson et al. 2010). There is thus an important need to understand how biodiversity components may be affected by fire frequency in order to assess species vulnerability and implement conservation action.

\section{Materials and methods}

Data

We searched the published literature for estimates of demographic parameters on reseeding and resprouting South African proteas (Table 1). We needed estimates of annual survival, reproduction and growth based on individuals followed trough time. Only few studies met this criterion and data were most complete for Protea neriifolia and P. repens (reseeders), and $P$. nitida (resprouter; Table 1). There was considerable variation among published estimates and we 
therefore chose the estimates that seemed to be most representative based on their sample size, analysis method and information given in the publications about the circumstances of data collection. Table 1 shows the values we used to parameterise the model described in the next section.

To examine whether our results depended on a particular choice of parameter value, we examined three alternative parameterisations, spanning the uncertainty in the values retrieved from the literature. The main uncertainties were around survival, growth and fecundity, which tend to be negatively correlated across plant life histories (Franco and Silvertown 2004). Our alternative parameterisations were one life history with high mortality and fast growth (survival reduced by $10 \%$ compared to baseline and growth increased by $100 \%$ ), one life history with high mortality and high fecundity (survival reduced by $10 \%$ and fecundity increased by $150 \%$ ), and one life history with high fecundity and slow growth (increased fecundity by $150 \%$ and decreased growth by $60 \%$ ). Details are given in Table S1.1 of Online Resource 1.

\section{Model description}

We modelled local population dynamics with a stochastic demographic model based on projection matrices. This model projects the number of individuals in each stage in year $t+1$ from the numbers in year $t$ :

$n_{t+1}=A_{e} n_{t}$

with

$A_{e}=\left(\begin{array}{ccccc}s_{1}(1-h) & 0 & f_{3} s_{1}(1-h) & f_{4} s_{1}(1-h) & f_{5} s_{1}(1-h) \\ s_{s} h & s_{2}\left(1-g_{23}\right) & f_{3} s_{s} h & f_{4} s_{s} h & f_{5} s_{s} h \\ 0 & s_{2} g_{23} & s_{3}\left(1-g_{34}\right) & s_{4} r_{4} & s_{5} r_{5} \\ 0 & 0 & s_{3} g_{34} & s_{4}\left(1-g_{45}\right)\left(1-r_{4}\right) & 0 \\ 0 & 0 & 0 & s_{4} g_{45} & s_{5}\left(1-r_{5}\right)\end{array}\right)$

Here, $n$ is a vector with the number of individuals in each stage and $A_{e}$ is the projection matrix where the subscript $e$ denotes that its entries depend on the environment, i.e. whether or not there is a fire. Fire happens after seed development in the annual cycle. We distinguish five stages: (1) seeds, (2) small vegetative plants, and (3-5) three size classes of reproductive plants. Plants (or seeds) in stage $x$ survive with probability $s_{x}$, while survival of seedlings during their first year is $s_{s}$. The growth rate from stage $x$ to stage $y$ is $g_{x y}$, and $f_{x}$ is the fecundity (number of viable seeds produced) in stage $x$. Seeds germinate after fire with probability $h$ ( $h=0$ in non-fire years), and resprouting adult plants are reset to the smallest adult stage (3) in fire years with probability $r_{x}$ ( $r=0$ in non-fire years). In fire years, we further multiplied $s$ by a stage-specific mortality factor $\left(1-m_{x}\right)$, where $m_{x}$ was 1 for reseeders (all reseeders die during fires).
Reproduction occurs shortly after the census at the beginning of the projection interval, which we take to be 1 year. We consider serotinous species that keep all seeds on the plant until the next fire, when they are dropped and germinate if there was a fire. The elements of the projection matrix $A_{e}$ depend on whether or not there is a fire in a particular year. We examine two plant types, a reseeder and a resprouter. The reseeder dies in a fire, whereas the resprouter survives either in its present state or as an underground tuber that produces a small adult plant in the following year. Seeds of both types germinate only after fires, and they start producing seeds after 2 years at the earliest. Figure 1 shows graphs of the life cycles of both types during fire-free years and years with fire. The transitions between life-stages are composed of survival rates, the probability of growing to the next stage (or being reduced to small adults in the case of resprouters during fire), and reproduction.

The sequence of fire and no-fire years was partly a firstorder Markovian process in that a fire year was always followed by a non-fire year. During the first year after fires, there is almost never enough biomass available to support another fire. In years after that, we modelled fires as occurring with a fixed probability $p$, independent of the preceding year. We explore dynamics for $p$ ranging from 0 to 1 . If $p=0$, there are no fires at all, and if $p=1$, fire and no-fire years alternate. Note that as $p$ approaches 0 or 1, the environment becomes deterministic. As $p$ goes from 0 to 1 , the temporal autocorrelation goes from 0 to -1 (Tuljapurkar 1997). We present the results in terms of fire-return

intervals, $(1 / p)+1$, in order to make them more comparable to earlier studies.

We chose to model fire-return intervals using fixed annual fire probabilities, because this introduced stochasticity in similar ways at all fire-return intervals that we considered, and therefore did not confound the effects of stochasticity and fire-return intervals. In reality, fires may become more likely with time since the last fire, which would lead to more regular fire-return intervals leading in the extreme case to a deterministic fire cycle with a given length. We re-ran our models with a deterministic fire cycle and all results remained qualitatively the same.

We used simulations to calculate the stochastic population growth rate $\log \lambda_{\mathrm{s}}$ and its sensitivity to changes in lifehistory parameters (Morris and Doak 2002). If viewed as a property of genotypes, $\lambda_{\mathrm{s}}$ is a measure of fitness (Metz 

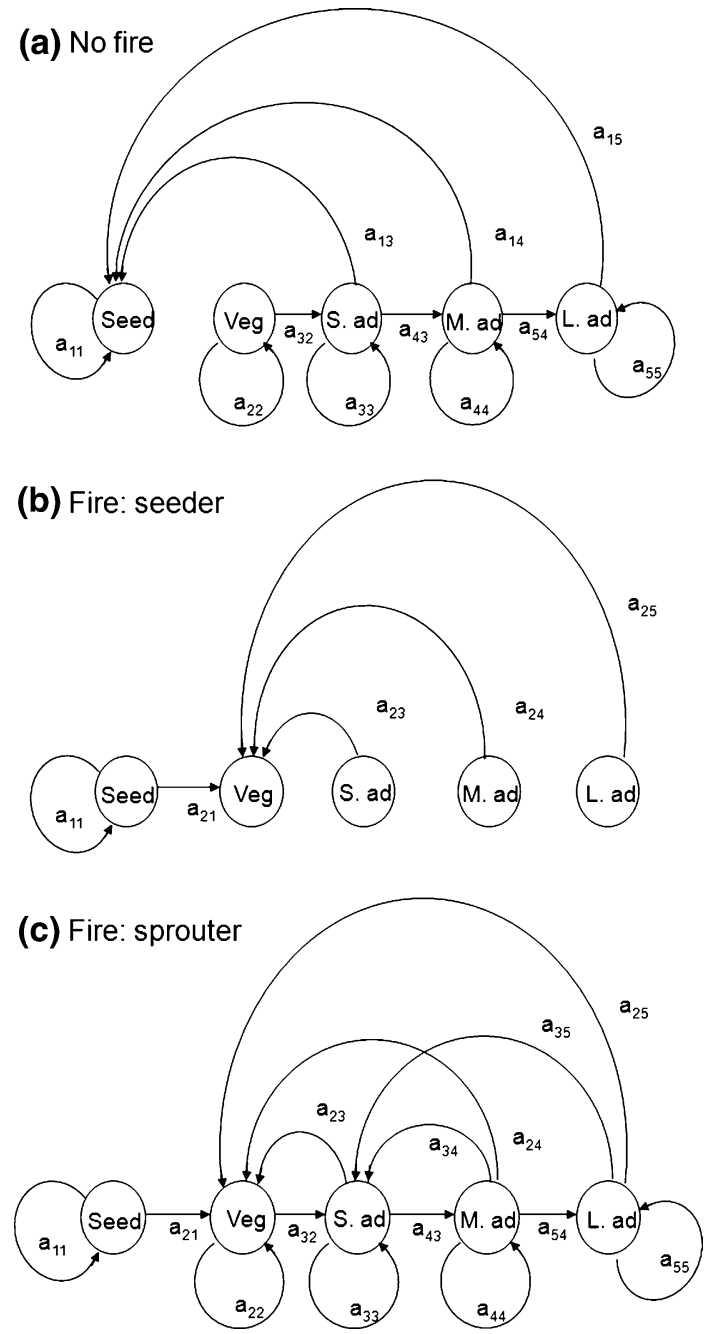

Fig. 1 Life-cycle graphs showing the possible life-history transitions during a year with a no fire (for both reseeders and resprouters), b reseeders during a year with fire, and $\mathbf{c}$ resprouters during a year with fire. The graphs illustrate the model developed in Eqs. 1 and 2 and the transitions $a_{x y}$ refer to the element in row $\mathrm{x}$ and column y of matrix A (Eq. 2). Life stages are: seeds, vegetative (Veg), small adult $(S . a d)$, medium adult (M.ad) and large adult $(L . a d)$ for reseeder and resprouter life-histories. Reseeders all die during fires, whereas resprouters can survive in their present state or be reset to the small adult stage. Seed production occurs at the beginning of each projection interval. Transitions $a_{23}, a_{24}$, and $a_{25}$ account for post-fire recruitment from seeds that were produced earlier in the same year

et al. 1992) and the sensitivities are equivalent to selection gradients (Van Tienderen 2000). We started the simulations with total population size $N_{0}=1$. This vector was left-multiplied 10,000 times by $\mathrm{A}_{e}$. After discarding the first 1,000 steps to eliminate transient dynamics (which generally disappeared with the first fire), we calculated the stochastic population growth rate as,

$\log \hat{\lambda}_{s}=\frac{1}{9000} \sum_{t=1001}^{10000} \log \left(\frac{N_{t+1}}{N_{t}}\right)$

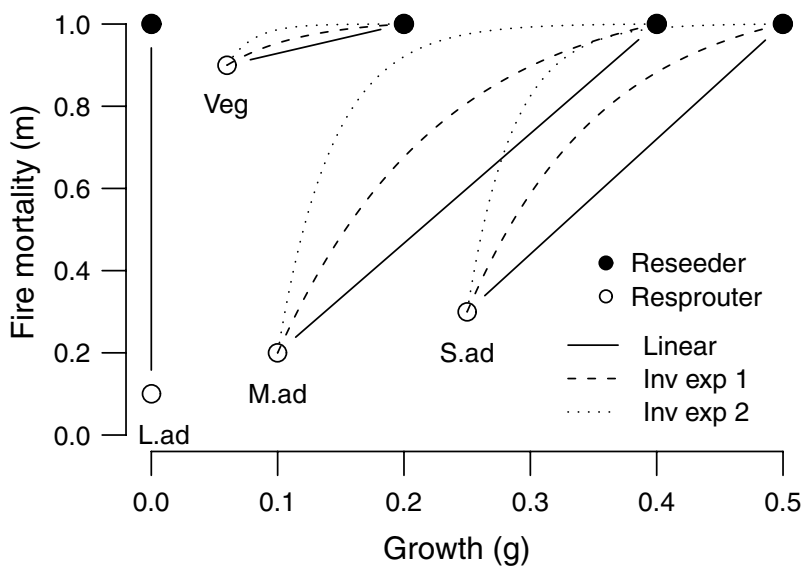

Fig. 2 Three hypothesized trade-offs between growth $(g)$ and fire mortality $(m)$ in four life stages. Open and filled circles represent resprouters and reseeders, respectively. The connecting lines represent the parameter values for intermediate life histories along a continuum between the two extremes and define the fire response strategies explored in Fig. 4

$N_{t}$ is the total population size at time $t$. In the next step we in turn changed each life-history parameter by 0.01 one at a time, and repeated the above step to calculate the altered stochastic population growth rate $\left(\log \hat{\lambda}_{s}^{\text {alt }}\right)$. While doing so, we retained exactly the same sequence of fire and non-fire years so that the life-history parameter was the only thing that changed among simulations. The sensitivity of the stochastic population growth rate to changes in the life-history parameter $a_{i}$ was then calculated as,

$S\left(a_{i}\right)=\frac{\hat{\lambda}_{s}^{\text {alt }}-\hat{\lambda}_{s}}{0.01}$

These steps were repeated for each life-history parameter of both reseeders and resprouters. Sensitivities are concerned with the effect of absolute changes in life-history parameters on $\lambda_{s}$, and it may therefore be difficult to compare two lifehistory parameters that are measured on different scales. Therefore, we also calculated elasticites (DeKroon et al. 1986 ) as the change in $\lambda_{\mathrm{s}}$ caused by a change of $1 \%$ in each life-history parameter. It has to be kept in mind, however, that the elasticities are dependent on the exact parameterisation of the model. For example, had we equivalently parameterised our model in terms of mortality rather than survival, we would have obtained different estimates for the elasticities but not for the sensitivities (Morris and Doak 2002). All calculations were done in R ( $\mathrm{R}$ Development Core Team 2012) and the code can be found in Online Resource 2.

Fire response streategy on a reseeder-resprouter continuum

Our main goal was to examine the fitness consequences of the trade-off between growth and mortality during fires (Bond and Midgley 2003). We considered three different 
forms of this trade-off, motivated by mechanistic considerations. First, we consider a linear relationship between growth and fire mortality (Fig. 2). However, a linear loss of protection (in terms of survival) as a function of decreased investment (and thus improved growth) is unlikely because the trade-off results from the effectiveness of protective tissue. It is more realistic to assume that protection will only become effective near the maximum level of investment, i.e. halving the thickness of protective tissue would result in less than half the survival benefits. We describe this type of trade-off using two curved relationships (Fig. 2). Using the model and methods described in the previous section, we calculated the fitness that intermediate forms would have achieved under different fire regimes. We started at the parameter values of resprouters (open symbols in Fig. 2), and examined 100 intermediate forms along the trade-off lines, up to the values for reseeders (closed symbols in Fig. 2).

\section{Event dependence versus interval dependence}

The characteristics of a particular fire event may be more important than the time between fire events (termed 'event dependence'; Bond and Van Wilgen 1996). We explored the generality of our results by varying two characteristics of fires that are important for plant population ecology: fire intensity and the season in which the fire happens (Bond and van Wilgen 1996). We used a simple extension of our model to accommodate each of these two effects in turn.

High intensity fires can kill resprouters that would normally survive. In addition to the two states, no fire and fire, we defined a third state, high-intensity fire, with its own version of projection matrix $A_{e}$. In high-intensity fires, mortality of resprouters either doubled or the fire killed all resprouters. In our model, high-intensity fires had the same effect on reseeders as normal intensity fires: they killed all plants. We ran the stochastic simulations as explained above, with a mean fire-return interval of 10 years. However, the fires now consisted of two types: normal intensity (identical to our previous simulations) or high intensity under a variety of fixed proportions. This approach allowed us to control the frequency of high-intensity fires but makes the unrealistic assumption that the probability of a fire being of high intensity is independent of the time since the last fire. We therefore explored an alternative parameterisation where the odds of a high-intensity fire linearly depended on time since the last fire (Online Resource 3).

Fires occurring during an unusual time of the year have two effects: they can kill the plants before the new generation of seeds is formed, and when many of the previous season's seeds have died; or they can lead to seed release at a time that is unfavourable for germination. The latter

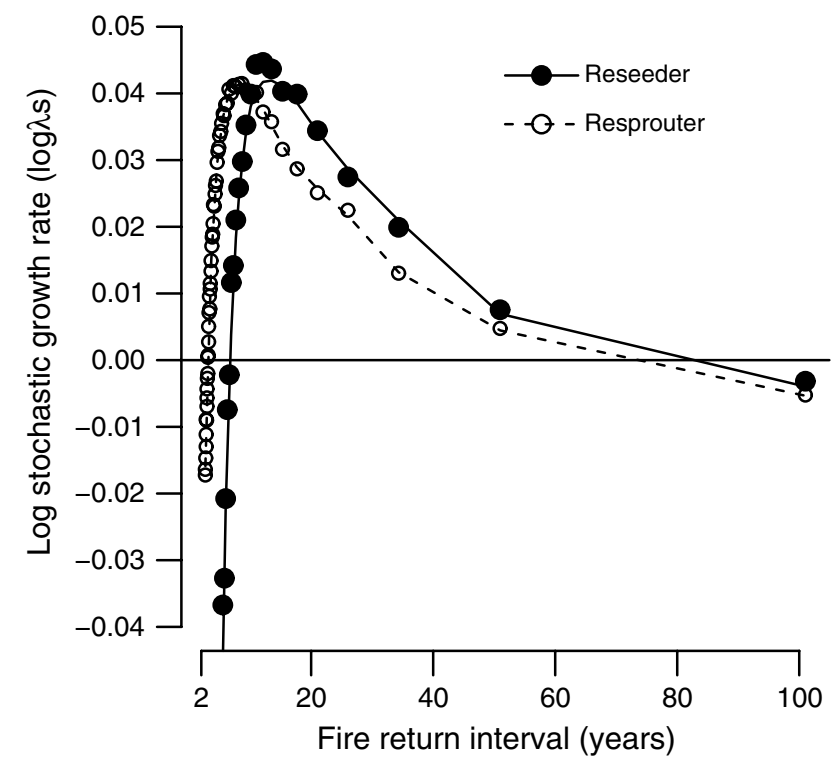

Fig. 3 Projected stochastic growth rate $\left[\log \left(\lambda_{\mathrm{s}}\right)\right]$ of reseeder and resprouter populations in relation to fire-return interval. A fire year was always followed by a year without fire, and fire with probability $p$ in years thereafter. Populations are stable at $\log \lambda_{\mathrm{s}}=0$, decline for negative values and increase for positive ones

situation also applies for fires during the normal season if they are followed by an unusual drought during the season normally favourable for germination and establishment. In both cases, the number of successful recruits will decrease. We ran the stochastic model as described in the section on model development, with a mean fire-return interval of 10 years. However, we simulated two different types of fire, normal fires and fires followed by reduced recruitment under a variety of fixed proportions. In the latter type of fire, we assumed $90 \%$ reduction in seeds released as a proxy for reduced recruitment.

\section{Results}

Analysing the basic model: responses of fitness parameters to differences in fire-return intervals

Both reseeder and resprouter populations declined at very short and very long fire-return intervals (Fig. 3), and our model thus realistically reproduces the dependency of these two life forms on fire. In our model, the optimal fire-return interval for maximum population growth (and thus fitness) of reseeders was 13 years, and 7 years for resprouters (Fig. 3). Reseeders did better than resprouters at firereturn intervals longer than 9 years. There were fire-return intervals where only resprouter populations achieved positive growth. At the chosen parameter values, this happened between 4 and 7 years. 
Table 2 Sensitivity and elasticity of the stochastic population growth rate $\left(\log \lambda_{\mathrm{s}}\right)$ to changes in the life-history parameters at fire-return intervals $(F R I)$ of 7 and 26 years

\begin{tabular}{|c|c|c|c|c|c|c|c|c|}
\hline & \multicolumn{4}{|c|}{ Sensitivity } & \multicolumn{4}{|l|}{ Elasticity } \\
\hline & \multicolumn{2}{|l|}{ Reseeder } & \multicolumn{2}{|c|}{ Resprouter } & \multicolumn{2}{|l|}{ Reseeder } & \multicolumn{2}{|c|}{ Resprouter } \\
\hline & $\mathrm{FRI}=7$ & $\mathrm{FRI}=26$ & $\mathrm{FRI}=7$ & $\mathrm{FRI}=26$ & $\mathrm{FRI}=7$ & $\mathrm{FRI}=26$ & FRI $=7$ & $\mathrm{FRI}=26$ \\
\hline Seed survival in seed bank & 0.121 & 0.058 & 0.068 & 0.039 & 0.049 & 0.023 & 0.026 & 0.015 \\
\hline Seedling survival & 2.773 & 0.725 & 1.068 & 0.467 & 0.140 & 0.035 & 0.051 & 0.023 \\
\hline Survival vegetatives & 0.310 & 0.147 & 0.208 & 0.193 & 0.297 & 0.135 & 0.190 & 0.179 \\
\hline Survival small adults & 0.163 & 0.071 & 0.251 & 0.108 & 0.161 & 0.067 & 0.237 & 0.103 \\
\hline Survival medium adults & 0.129 & 0.081 & 0.297 & 0.203 & 0.128 & 0.077 & 0.279 & 0.195 \\
\hline Survival large adults & 0.218 & 0.698 & 0.241 & 0.530 & 0.216 & 0.663 & 0.227 & 0.508 \\
\hline Growth from vegetative to small adult & 0.554 & 0.072 & 0.743 & 0.232 & 0.112 & 0.014 & 0.043 & 0.014 \\
\hline Growth from small to medium adult & 0.067 & 0.008 & 0.071 & 0.014 & 0.034 & 0.004 & 0.017 & 0.003 \\
\hline Growth from medium to large adult & 0.033 & 0.006 & 0.106 & 0.032 & 0.013 & 0.002 & 0.010 & 0.003 \\
\hline Fecundity small adults & 0.004 & 0.000 & 0.001 & 0.000 & 0.054 & 0.003 & 0.008 & 0.001 \\
\hline Fecundity medium adults & 0.001 & 0.000 & 0.001 & 0.000 & 0.039 & 0.004 & 0.021 & 0.005 \\
\hline Fecundity large adults & 0.001 & 0.000 & 0.000 & 0.000 & 0.059 & 0.032 & 0.025 & 0.018 \\
\hline Germination rate after fire & 0.116 & 0.039 & 0.054 & 0.025 & 0.115 & 0.037 & 0.051 & 0.024 \\
\hline Germination rate no fire & 0.395 & 2.423 & 0.208 & 1.090 & 0.000 & 0.000 & 0.000 & 0.000 \\
\hline Fire mortality vegetatives & & & -0.039 & -0.005 & & & -0.034 & -0.004 \\
\hline Fire mortality small adults & & & -0.044 & -0.003 & & & -0.013 & -0.001 \\
\hline Fire mortality medium adults & & & -0.051 & -0.005 & & & -0.010 & -0.001 \\
\hline Fire mortality large adults & & & -0.033 & -0.009 & & & -0.003 & -0.001 \\
\hline Probability being reset during fire: medium adults & & & -0.006 & 0.000 & & & -0.003 & 0.000 \\
\hline Probability being reset during fire: large adults & & & -0.015 & -0.003 & & & -0.007 & -0.001 \\
\hline
\end{tabular}

Sensitivity and elasticity for fire mortality are both negative because the increase mortality rate decreases population growth. See Online Resource 4 for more detailed sensitivity analyses

Sensitivity estimates measure selection strength as the effect of small absolute changes in life-history parameters on fitness $\left(\lambda_{\mathrm{s}}\right)$, and we expected these estimates to depend on the length of the fire-return interval. We therefore repeated the sensitivity analysis for fire-return intervals between 2 and 35 years (see Figures S4.1-S4.2 of Online Resource 4 for details) and found similar patterns in reseeders and resprouters: reproduction and survival of early life stages were relatively more important at short fire-return intervals, whereas adult survival was relatively more important at long fire-return intervals.

Focusing on a relatively short fire-return interval of 7 years (Table 2), fitness was most sensitive to changes in seedling survival and growth from the vegetative to small adult life stage. At long fire-return intervals (e.g. 26 years; Table 2), fitness was most sensitive to changes in the germination rate during years with no fire, and to survival of seedlings and large-adults, whereas growth, fecundity, and fire mortality (in the case of resprouters) were less important.

Survival and reproduction are measured on different scales and sensitivity estimates between the two types of life-history parameters may not be strictly comparable. We therefore also calculated elasticities, or changes in population growth in response to relative changes, in each life-history parameter. The elasticity analysis confirmed that fecundity was relatively less important for population growth than survival and growth (Table 2, and Figs. S4.3, S4.4).

Reseeders and resprouters were similar in their patterns of sensitvity and elasticity. However, overall, seedling survival was relatively more important in reseeders, and survival of all adult stages was relatively more important in resprouters, compared to the rest of the life-history parameters.

Above, we have followed Bond and Midgley (2003) who found (in their Fig. 2) that reseeders and resprouters among South African proteas (genus Leucadendron) produced similar numbers of seeds given a certain plant size. However resprouters may produce fewer seeds than comparable reseeder species (Lamont 1985; Bond and van Wilgen 1996; Verdu 2000; Higgins et al. 2008). Halving seed production of resprouters compared to reseeders in our model, the maximum fitness $\lambda_{\mathrm{s}}$ of resprouters decreased from 0.041 to 0.013 and the optimal fire interval increased from 7 to 11 years. 
Fire response streategy on a reseeder-resprouter continuum

We examined three different shapes of the trade-off between fire mortality and growth (Fig. 2) and calculated the expected fitness of intermediate strategies on those lines. Regardless of the shape of trade-off between fire mortality and plant growth rates, the fire-return interval had a stronger effect on population growth than the position on the reseeder-resprouter continuum (Fig. 4, shown by the steeper slopes in the direction of the $x$-axis, i.e. the fire-return interval). Resprouters achieved their highest population growth rates at shorter fire-return intervals than reseeders, confirming our results above. The fitness of intermediate forms depended on the shape of the trade-off. With a linear relationship between growth and fire mortality, intermediate forms tended to achieve slightly higher population growth rates than pure reseeders and resprouters, and the maximum was at $63 \%$ reseeder (Fig. 4a). With an inverse exponential relationship between growth and fire mortality, intermediate forms performed worse than pure reseeders and resprouters (Fig. 4b, c). As the non-linearity in the trade-off becomes stronger, a clear saddle appears in the fitness surface and fire-return intervals between $\sim 10$ and $\sim 20$ years exert selective pressure to specialise towards the ends of the reseeder-resprouter continuum (Fig. 4c). These patterns were similar for the three alternative parameterisations (Figures S1.1-S1.3 in Online Resource 1) showing that these results were true across a set of different life histories.

\section{Event dependence versus interval dependence}

We examined the effect of occasional high-intensity fires on fitness of the reseeders and resprouters. Both regular and high-intensity fires killed all reseeders, and the proportion of high-intensity fires therefore had no effect on the reseeders' fitness in our model (Fig. 5). High-intensity fires also had little effect on resprouters if they led to twice the mortality of normal fires. If high-intensity fires killed all resprouters, however, resprouter fitness was depressed below 0 when $40 \%$ or more of the fires were of high intensity. These results remained qualitatively the same if we modelled the probability of a high-intensity fire as a function of the time since the last fire (Online Resource Fig. S3.2), and are consistent with our sensitivity analysis showing that fitness was relatively insensitive to fire mortality (Table 2).

We obtained these results under the assumption that reseeders and resprouters produce a similar number of seeds given a certain size class. However, resprouters may produce relatively fewer seeds than reseeders (Bond and Midgley 2003; Higgins et al. 2008). In that case, the curves in Fig. 5 would be shifted down for resprouters, and we

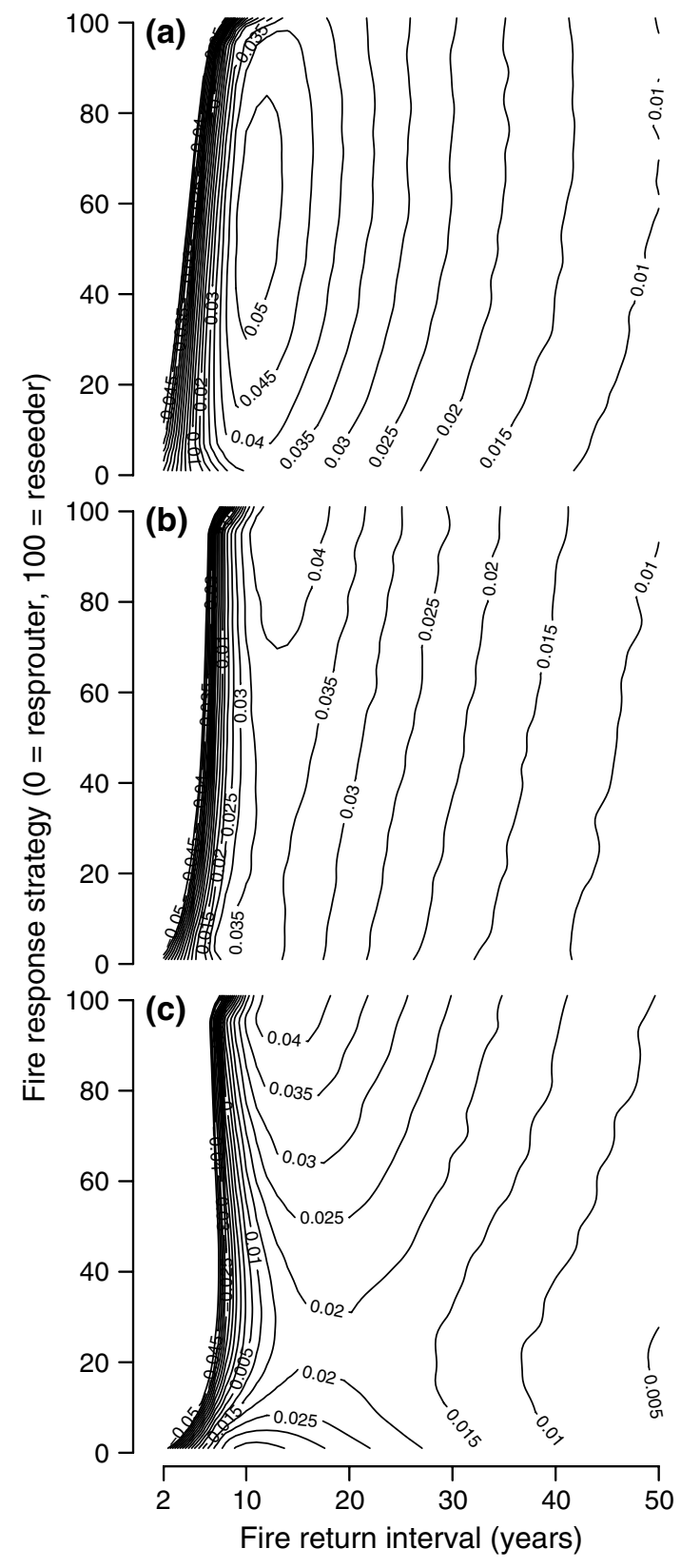

Fig. 4 Fitness landscape (contours are lines of equal fitness, measured as $\log$ stochastic growth rate $\lambda_{\mathrm{s}}$ ) in relation to fire-return interval and fire response strategy. The latter is defined as a continuum from resprouter $(0)$ to reseeder (100) with intermediate parameter values for growth and fire mortality as depicted by the lines connecting the values for the extreme resprouter and reseeder life histories in Fig. 2. The panels show fitness landscapes under the three hypothesized trade-offs between fire mortality and growth: a linear, b, c inverse exponential trade-offs

would predict that resprouter populations decline at lower proportions of high-intensity fires.

Aseasonal fires reduced fitness in both reseeders and resprouters (Fig. 6). However, the effect was larger on reseeders than on resprouters. Again, this is consistent with our 


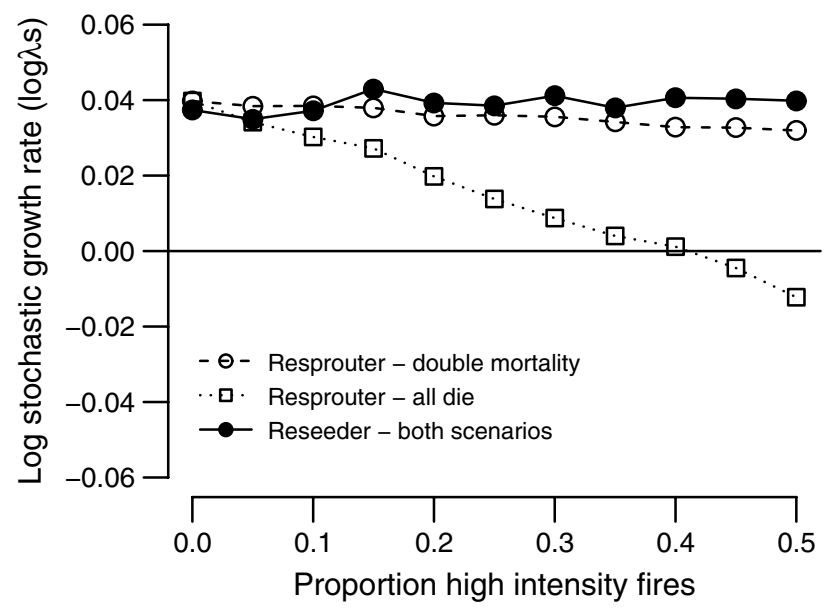

Fig. 5 Effects of high-intensity fires on reseeder and resprouter population growth. Average fire-return interval was 10 years, but the proportion of high-intensity fires varied from 0 to $50 \%$. We assumed high-intensity fires doubled mortality compared to normal intensity fires (open circles) or killed all individuals (open squares). Since reseeders all die during any fire, fire intensity did not affect their population growth rate

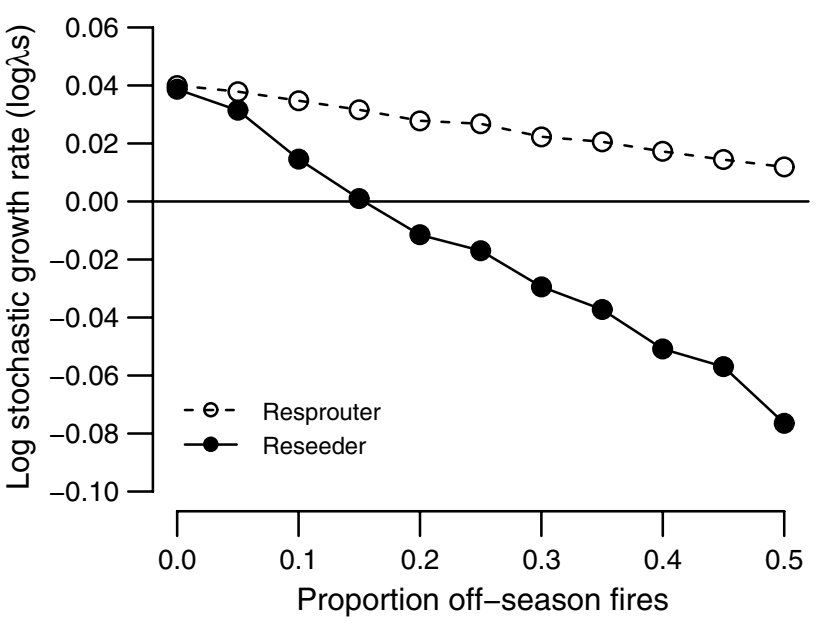

Fig. 6 Effects of aseasonal fires on reseeder and resprouter population growth. Average fire-return interval was 10 years, but the proportion of off-season fires varied from 0 to $50 \%$. Off-season fires reduced seed release by $90 \%$ in both resprouters and reseeders

sensitvity analysis showing that seedling survival is relatively more important in reseeders than in resprouters (Table 2) and is confirmed in the literature (e.g. Bond et al. 1984).

\section{Discussion}

In fire-prone Mediterranean-type plant communities, two co-existing growth strategies differ in their response to fire (Le Maitre 1992; Higgins et al. 2008; Keeley et al. 2012). Reseeders die and recruit from seeds whereas resprouters generally survive and resprout from protected buds or tubers. The strategies essentially differ in how they allocate resources to fast growth or protective tissue (Le Maitre 1992; Bond and Midgley 2003). The two distinct strategies are found in all major plant families living in fire-prone habitats with very few intermediate forms (Schutte et al. 1995; Ojeda 1998). Apparently these habitats select for the evolution of two coexisting specialist forms rather than an intermediate strategy, but the reason for why this should be so is unclear. The phylogenetic evidence clearly shows that transition from one strategy into the other have been made repeatedly during evolutionary time (Schutte et al. 1995; Bond and Midgley 2003; Linder 2003; Barker et al. 2004). However, it is still unclear how such transitions in fire biology occur (Linder 2003).

Using a demographic model parameterised from published data on life-history parameters of resprouting and reseeding Protea species from the Cape Floristic Region, we found that the fire-return interval affected fitness differently in the two strategies with resprouters being able to persist at shorter fire-return intervals. These results are consistent with field observation showing that reseeders are more frequent at low fire frequencies whereas resprouters are dominant at higher fire frequencies (Le Maitre and Midgley 1992; Ojeda 1998; Vlok and Yeaton 2000; Lamont et al. 2011). Our results also confirm earlier work showing that fire can be a strong structuring force in plant communities (Higgins et al. 2000a).

The length of the fire-return interval also affected the strength of selection, measured as the sensitivity of the population growth rate to changes in life-history parameters. With decreasing fire-return intervals, seedling survival and growth became more important because quick establishment is critical under these circumstances. Under long fire-return intervals, the sensitivity analysis suggested that an ability to germinate between fires could be strongly advantageous. Germination between fires is uncommon in the South African fynbos and the Australian kwongan but can be common in other Mediterranean-type regions (Keeley et al. 2012). Based on our results, we predict that the maintenance of serotiny depends on medium fire-return intervals (Le Maitre 1992).

Our results provide a possible explanation for the observation of the striking dominance of Mediterranean climate plant communities by reseeders and resprouters, with an apparent absence of intermediate forms, but with a frequent evolutionary switch between the two strategies. Our demographic model showed that there are two distinct fitness peaks at these extreme life-history strategies, when the trade-off between fire mortality and growth is concave-down. This matches the pattern that we observe among Protea where the majority of species are reseeders or resprouters, with very few intermediate forms, i.e. 
facultative resprouters (e.g. some Fabaceae, Schutte et al. 1995, and Ericaceae, Ojeda 1998). If the trade-off between growth and fire mortality is well described by an inverse exponential function, our results show that the length of the fire-return interval can affect the outcome of evolution, and support the notion that long fire-return intervals would favour the evolution of reseeders and short fire-return intervals favour resprouters (Keeley 1977; Barker et al. 2004). For example, Kraaij et al. (2013) found that a reseeding protea failed to reproduce with a 5-year fire-return interval. Along a gradient of fire frequency, the fitness peaks were relatively close together and separated only by a narrow fitness trough (Fig. 4b, c). This result could explain why selection leads to one of the two strategies and why the two strategies are phylogenetically labile if fire-return intervals vary around 7-25 years, as observed in these ecosystems. If the trade-off between fire mortality and growth is linear, then an intermediate strategy would be optimal (Fig. 4a), and a dominance of intermediate forms would be expected. This is not supported by observation of life-history data in the fynbos (Schutte et al. 1995; Ojeda 1998). A non-linear trade-off is expected if it is mediated by the function of protective tissue, such as bark. Growing bark of half the required thickness to withstand crown fires, for example, would incur half the cost but buy less than half the protection. Our results thus suggest that the nature of this tradeoff is important for understanding the evolution of reseeders and resprouters.

Within the fynbos biome, fire regimes are often managed to aim for a 15-year fire-return interval, but intervals can vary widely between less than 10 years and more than 20 years. According to our model, both reseeders and resprouters should be able to persist under such a regime. However, frequent off-season fires, or fires not followed by favourable conditions for establishment, including unpredictable rainfall, favoured resprouters. On the other hand, high-intensity fires favoured reseeders, especially if resprouters produce less seed than reseeders. In that case, populations of resprouters would be sensitive to the occurrence of occasional intense fires, with only one intense fire in 100 years resulting in negative population growth of resprouters in our simulations. The frequency of intense fires could well increase with likely climate change scenarios forecast for the fynbos (Wilson et al. 2010).

Our model could be extended in several ways. First, it does not account for demographic stochasticity and is not yet suitable to determine extinction risk. Reseeders possibly carry a higher risk of local population extinction at each fire event than resprouters, as they risk an entire generation's reproductive effort on a single recruitment event (Higgins et al. 2000b; Ojeda et al. 2005). Our main result, i.e. the general shape of the fitness landscape should not be affected by demographic stochasticity but it would be important for projecting extinction risk, for example under different scenarios for fire management.

The model also does not account for genetic effects and senescence. Resprouters with longer generation times and with over-lapping generations may be more susceptible to build-up of somatic mutations (Lamont et al. 2011) and consequent genetic load (Keeley et al. 2012), whereas the shorter and non-overlapping generations of reseeders may increase their scope for adaptive evolutionary response. We do not expect that these effects would change our results in a qualitative way.

Our main results were robust to relatively large changes in parameter values, and the conclusions held across four different model parameterisations that span a wide range of the plausible parameter space and accounted for likely correlations between traits. The results were also robust to additional mortality due to high-intensity fires and reduced recruitment due to unfavourable conditions after the fire. It would be interesting to model the interplay between lifehistory trade-offs and environmental factors more explicitly and see to what extent the environment can explain patterns of dominance of the two strategies within and among Mediterranean-type ecosystems around the globe. In this regard, our model complements earlier work that has focused on competition and co-existence between the two strategies (Ojeda et al. 2005; Higgins et al. 2008).

Acknowledgments We thank William Bond, Tony Rebelo, Ted Oliver, Anne-Lise Schutte Vlok and two anonymous reviewers for helpful comments and discussions. This study was funded by the South African National Research Foundation. The NRF accepts no liability for opinions, findings and conclusions or recommendations expressed in this publication. This study complies with the current laws of South Africa.

Conflict of interest The authors declare that they have no conflict of interest.

\section{References}

Barker NP, Vanderpoorten A, Morton CM, Rourke JP (2004) Phylogeny, biogeography, and the evolution of life-history traits in Leucadendron (Proteaceae). Mol Phylogenet Evol 33:845-860. doi:10.1016/j.ympev.2004.07.007

Barraclough TG (2006) What can phylogenetics tell us about speciation in the Cape flora? Divers Distrib 12:21-26. doi:10.1111/j.1366-9516.2006.00208.x

Bond WJ, Keeley JE (2005) Fire as a global "herbivore": the ecology and evolution of flammable ecosystems. Trends Ecol Evol 20:387-394

Bond WJ, Midgley JJ (1995) Kill thy neighbor-an individualistic argument for the evolution of flammability. Oikos 73:79-85

Bond WJ, Midgley JJ (2001) Ecology of sprouting in woody plants: the persistence niche. Trends Ecol Evol 16:45-51

Bond WJ, Midgley JJ (2003) The evolutionary ecology of sprouting in woody plants. Int J Plant Sci 164:S103-S114 
Bond WJ, van Wilgen BW (1996) Fire and plants. Chapman and Hall, London

Bond WJ, Vlok JHJ, Viviers M (1984) Variation in seedling recruitment of Cape proteaceae after fire. J Ecol 72:209-221

Coetzee JH, Giliomee JH (1987) Seed predation and survival in the infructescences of Protea repens (Proteaceae). S Afr J Bot 53:61-64

Cowling RM (1987) Fire and its role in coexistence and speciation in Gondwanan shrublands. S Afr J Sci 83:106-112

Cowling RM (1992) The ecology of fynbos: nutrients, fire and diversity. Oxford University Press, Cape Town

Cowling RM, Holmes PM, Rebelo AG (1992) Plant diversity and endemism. In: Cowling RM (ed) Ecology of fynbos: nutrients, fire and diversity. Oxford University Press, Cape Town, pp 62-112

DeKroon H, Plaisier A, Vangroenendael J, Caswell H (1986) Elasticity: the relative contribution of demographic parameters to population growth rate. Ecology 67:1427-1431

Enright NJ, Marsula R, Lamont BB, Wissel C (1998a) The ecological significance of canopy seed storage in fire-prone environments: a model for non-sprouting shrubs. J Ecol 86:946-959. doi:10.1046/j.1365-2745.1998.00312.x

Enright NJ, Marsula R, Lamont BB, Wissel C (1998b) The ecological significance of canopy seed storage in fire-prone environments: a model for resprouting shrubs. J Ecol 86:960-973. doi:10.1046/j.1365-2745.1998.00312.x

Franco M, Silvertown J (2004) Comparative demography of plants based upon elasticities of vital rates. Ecology 85:531-538

Goldblatt P, Manning JC (2002) Plant diversity of the Cape region of southern Africa. Ann Mo Bot Gard 89:281. doi:10.2307/3298566

Higgins SI, Bond WJ, Trollope WSW (2000a) Fire, resprouting and variability: a recipe for grass-tree coexistence in savanna. J Ecol $88: 213-229$

Higgins SI, Pickett STA, Bond WJ (2000b) Predicting extinction risks for plants: environmental stochasticity can save declining populations. Trends Ecol Evol 15:516-520

Higgins SI, Flores O, Schurr FM (2008) Costs of persistence and the spread of competing seeders and sprouters. J Ecol 96:679-686. doi: $10.1111 / \mathrm{j} .1365-2745.2008 .0$

Keeley JE (1977) Seed production, seed populations in soil, and seedling production after fire for two congeneric pairs of sprouting and non-sprouting chaparral shrubs. Ecology 58:820-829

Keeley JE, Zedler PH (1978) Reproduction of chaparral shrubs after fire-comparison of sprouting and seeding strategies. Am Midl Nat 99:142-161

Keeley JE, Bond WJ, Bradstock RA, Pausas JG, Rundel PW (2012) Fire in Mediterranean ecosystems: ecology, evolution and management. Cambridge University Press, Cambridge

Kraaij T, Cowling RM, van Wilgen BW, Schutte-Vlok A (2013) Proteaceae juvenile periods and post-fire recruitment as indicators of minimum fire return interval in eastern coastal fynbos. Appl Veg Sci 16:84-94. doi:10.1111/j.1654-109X.2012.01209.x

Lamont BB (1985) The comparative reproductive biology of three Leucospermum species (Proteacea) in relation to fire responses and breeding system. Aust J Bot 33:139-145

Lamont BB, Enright NJ, He T (2011) Fitness and evolution of resprouters in relation to fire. Plant Ecol 212:1945-1957. doi:10.1007/s11258-011-9982-3

Le Maitre DC (1987) Dynamics of canopy-stored seed in relation to fire. In: Cowling RM, Le Maitre DC, McKenzie B, Prys-Jones RP, Van Wilgen BW (eds) Disturbance and the dynamics of fynos communities. Foundation for Research and Development (CSIR), Pretoria, pp 24-45

Le Maitre DC (1992) The relative advantages of seedling and sprouting in fire-prone environments: a comparison of life histories of Protea neriifolia and Protea nitida. In: van Wilgen BW, Richardson DM, Kruger FJ, van Hensbergen HJ (eds) Fire in South
African mountain fynbos: ecosystems, community and species response at Swarboskloof. Springer, New York, pp 123-144

Le Maitre DC, Midgley JJ (1992) Plant reproductive ecology. In: Cowling RM (ed) The ecology of fynbos: nutrients, fire and diversity. Oxford University Press, Cape Town, pp 135-174

Linder HP (2003) The radiation of the Cape flora, southern Africa. Biol Rev Camb Philos Soc 78:597-638

Metz JAJ, Nisbet RM, Geritz SAH (1992) How should we define "fitness" for general ecological scenarios? Trends Ecol Evol 7:198-202

Morris WF, Doak DF (2002) Quantitative conservation biology. Sinauer, Sunderland

Musil CF (1991) Seed bank dynamics in sand plain lowland fynbos. S Afr J Bot 57:131-142

Ojeda F (1998) Biogeography of seeder and resprouter Erica species in the Cape Floristic Region-where are the resprouters? Biol J Linn Soc 63:331-347. doi:10.1111/j.1095-8312.1998.tb01521.x

Ojeda F, Brun FG, Vergara JJ (2005) Fire, rain and the selection of seeder and resprouter life-histories in firerecruiting, woody plants. New Phytol 168:155-165. doi:10.1111/j.1469-8137.2005.01486.x

Pausas JJG, Bradstock RAR, Keith DA, Keeley JEJ (2004) Plant functional traits in relation to fire in crown-fire ecosystems. Ecology 85:1085-1100. doi:10.1890/02-4094

Pratt RB, Jacobsen AL, Hernandez J, Ewers FW, North GB, Davis SD (2012) Allocation tradeoffs among chaparral shrub seedlings with different life history types (Rhamnaceae). Am J Bot 99:14641476. doi:10.3732/ajb.1200193

R Development Core Team (2012) R: a language and environment for statistical computing, 2.15.0. R Foundation for Statistical Computing, Vienna, Austria

Roff DA, Fairbairn DJ (2007) The evolution of trade-offs: where are we? J Evol Biol 20:433-447

Schutte AL, Vlok JHJ, Wyk B-E (1995) Fire-survival strategy-a character of taxonomic, ecological and evolutionary importance in fynbos legumes. Plant Syst Evol 195:243-259. doi:10.1007 /BF00989299

Stearns SC (1992) The evolution of life histories. Oxford University Press, Oxford

Takhtajan A (1986) Floristic regions of the world. University of California Press, Berkeley

Tuljapurkar S (1997) Stochastic matrix models. In: Tuljapurkar S, Caswell H (eds) Structured-population models in marine, terrestrial, and freshwater systems. Chapman and Hall, New York, pp 59-87

Van Tienderen PH (2000) Elasticities and the link between demographic and evolutionary dynamics. Ecology 81:666-679

Verdu M (2000) Ecological and evolutionary differences between Mediterranean seeders and resprouters. J Veg Sci 11:265-268

Vesk PA, Westoby M (2004) Sprouting ability across diverse disturbances and vegetation types worldwide. J Ecol 92:310-320

Vlok JHJ, Yeaton RI (1999) The effect of overstorey proteas on plant species richness in South African mountain fynbos. Divers Distrib 5:213-222. doi:10.1046/j.1472-4642.1999.00055.x

Vlok JHJ, Yeaton RI (2000) The effect of short fire cycles on the cover and density of understorey sprouting species in South African mountain fynbos. Divers Distrib 6:233-242. doi:10.1046/j.1472-4642.2000.00087.x

Wells PV (1969) Relation between mode of reproduction and extent of speciation in woody genera of California chaparral. Evolution 23:264-267

Wilson AM, Latimer AM, Silander JA Jr, Gelfand AE, de Klerk H (2010) A hierarchical Bayesian model of wildfire in a Mediterranean biodiversity hotspot: implications of weather variability and global circulation. Ecol Model 221:106-112. doi:10.1016/j.ecolm odel.2009.09.016 